\title{
e-Migrinter
}

$7 \mid 2011$

Réflexions croisées sur les migrations en Afrique de l'Ouest

\section{Face au terrain : sensibilités plurielles et adaptations singulières}

\section{Mickaël Quintard}

\section{OpenEdition}

\section{Journals}

Édition électronique

URL : https://journals.openedition.org/e-migrinter/917

DOI : 10.4000/e-migrinter.917

ISSN : 1961-9685

Éditeur

UMR 7301 - Migrinter

Édition imprimée

Date de publication : 1 septembre 2011

Pagination : 28-35

ISSN : 1961-9685

\section{Référence électronique}

Mickaël Quintard, «Face au terrain : sensibilités plurielles et adaptations singulières », e-Migrinter [En ligne], 7 | 2011, mis en ligne le 14 septembre 2017, consulté le 20 mai 2021. URL : http:// journals.openedition.org/e-migrinter/917 ; DOI : https://doi.org/10.4000/e-migrinter.917 
Face au terrain : sensibilités plurielles et adaptations singulières

\section{Mickaël Quintard}

D ans un récent travail de thèse présenté en décembre dernier (2010), Yann

Calbérac porte son regard sur la manière dont les géographes ont pris l'habitude d'interroger la place qu'occupe le terrain au sein de leurs démarches, ou plutôt celle qu'ils ont longtemps prise de ne pas la questionner. Il souligne notamment le paradoxe entre l'importance ancienne que revêt celui-ci ${ }^{1}$ dans la démarche et l'identité du géographe ${ }^{2}$, ainsi que le manque de réflexivité à son égard, l'impensé ${ }^{3}$ qu'il a longtemps pu constituer. Ce travail de thèse semble aujourd'hui participer à une certaine ré-interrogation de la pratique du terrain par les géographes.

\footnotetext{
"Envisagé comme une instance de validation et de légitimation des savoirs géographiques» [Calbérac Yann, $2006: 1]$.

2 De l'époque Vidalienne à aujourd'hui, en passant par l'époque de la « crise de la géographie »...

3 Aucun des géographes interrogés par Yann Calbérac n'a été en mesure de réellement définir la pratique du terrain.
}

Introduction

À ce titre, d'autres initiatives ont récemment vu le jour, comme la tenue du colloque (2008) « $\grave{A}$ travers l'espace de la méthode. Les dimensions du terrain en géographie ${ }^{4}$ » - lequel est au cœur de la démarche de Yann Calbérac - ou la nouvelle revue en ligne intitulée "Carnets de géographes», où la rubrique «Carnets de terrain» est exclusivement dédiée aux expériences de terrain, livrant l'impression de traduire un nouveau rapport, davantage décomplexé (Collignon, Retaillé, 2010), entre le terrain et les géographes. C'est dans cette veine que tentent de s'inscrire ces propos, en lien notamment avec certains évènements qui rendent aujourd'hui délicate la pratique du terrain dans certaines régions d'Afrique de l'Ouest. Nous saisissons ainsi cette instabilité comme une occasion de réinterroger des pratiques de recherches à travers le lien qui unit le géographe à son terrain.

Le but n'est pas ici de retracer les différentes significations qu'a pu avoir la pratique du terrain au cours du siècle dernier, ni d'interroger le silence des géographes sur leurs pratiques; mais davantage de questionner les différentes terminologies employées pour qualifier un terrain, qu'il s'agisse de celle de "terrain sensible », de «terrain miné » ou de «mise en danger ». La réflexion s'alimente d'une expérience de terrain vécue au Niger en 2010 et s'intéresse notamment aux répercussions que diverses difficultés de terrain peuvent engendrer.

\footnotetext{
${ }_{4}^{4}$ Arras, 18-20 juin 2008.
} 


\section{Question de sémantique}

Dans la littérature abordant certaines difficultés de terrain, il est souvent fait mention de l'expression "terrains sensibles » (Bouillon et al., 2006). Si le terme de «sensible» peut être envisagé, de manière assez simple, comme quelque chose que l'on doit traiter avec une certaine attention et une vigilance particulière, il convient de s'interroger sur le pourquoi de ces précautions. Il est possible d'établir beaucoup de distinctions et de nuances autour de l'expression "terrains sensibles », la question centrale étant de définir ce qui est jugé sensible. Ce terme fait-il référence à des lieux d'enquête (centres de rétention, "ghettos ", etc.), à des populations ou des conditions sociales (sans-papiers, SDF, etc.), à des enjeux politiques plus larges qui peuvent plus ou moins contraindre et conditionner le chercheur (relations avec le HCR dans les camps de réfugiés), ou s'agit-il plus simplement du sujet abordé dans des contextes et lieux déterminés (la question alimentaire dans des pays sous régime d'aide, etc.) ?

Si les situations sensibles peuvent ainsi être variées, la «sensibilité » peut également être «mouvante » et changer de nature. Ceci peut notamment être le cas lorsque c'est le sujet envisagé qui rend par ricochet le terrain sensible, la question alimentaire ne revêt par exemple pas partout la même importance, et n'est donc pas également «sensible» en chaque lieu. Cette problématique peut ainsi se poser avec plus d'acuité dans certains endroits que dans d'autres. Elle peut parfois être politiquement très sensible, notamment dans des pays comme le Niger où elle peut se trouver instrumentalisée au sein de stratégies d'attraction d'aides internationales. Ici, c'est parce que le sujet abordé est initialement sensible que le terrain, dans sa dimension spatiale, peut également le devenir.

À l'inverse, un terrain peut être «sensible» de manière complètement indépendante du sujet, notamment quand il est traversé par des situations d'instabilité politique et de guerres, ou encore de risques climatiques et sanitaires. Il est alors question des difficultés relatives à la pratique physique du terrain, voire de la mise en danger d'ordre corporel. Sous l'angle des ethnologues, Albeta Dionigi (2001) discute alors de l'expression «terrains minés » susceptible de caractériser des contextes de recherche présentant des difficultés d'ordre méthodologiques, physiques, épistémologiques ou symboliques. Dans le cas de la mise en danger physique, elle y souligne les connotations militaires qui accompagnent ces représentations: "Le terrain est un lieu d'affrontements, et "aller sur le terrain 》 signifie originellement se rendre sur un champ de bataille (...) Sur le terrain, l'ethnologue est coupé de son lieu "propre ». Il doit circuler avec ses propres moyens sur un territoire qu'il ne maîtrise pas, et qui est au contraire contrôlé par d'autres institutions, d'autres instances de pouvoir. Le terrain est donc pour l'ethnologue un champ d'opérations, selon la vieille tradition militaire » (Dionigi, 2001 : 6-7).

Nous voyons donc que le terme de «terrain sensible» correspond à plusieurs types de situations relativement différentes les unes des autres. Dès lors qu'il est envisagé de relater et d'interroger des difficultés liées à la pratique du terrain, la pluralité d'aspects de la sensibilité invite donc, sémantiquement, à se placer et à se justifier. En nous appuyant sur notre expérience de terrain, nous nous approprions les deux termes de «sensible» et "miné », et envisagerons le passage de l'un à l'autre. Le terme « sensible » renverra à la dimension spatiale et sera considéré à travers des difficultés de terrain pouvant être jugées comme «classiques » à l'anthropologie; celui de «miné» comme représentatif d'une élévation du niveau de sensibilité par rapport à une situation antérieure, conduisant non plus à des stratégies d'adaptation, mais de contournement, voire de remplacement. 


\section{Inquiétudes et difficultés de la méthode anthropologique en " terrain sensible "}

\section{Particularités « tropicales »}

Il convient de rappeler la place centrale qu'occupe la pratique du terrain dans les études géographiques sur l'Afrique. Comme le souligne Rolland Pourtier, «le terrain a toujours joué un rôle important pour les géographes effectuant des recherches dans les pays tropicaux » (2007 : 437). Cette place du terrain est d'autant plus centrale que dans un certain nombre de cas, le chercheur fait face à un manque de sources bibliographiques, ou à des problèmes de fiabilité ou même d'absence de certaines données statistiques. Le terrain est par conséquent l'outil principal du géographe intéressé par l'Afrique. Si l'on ajoute à ces quelques éléments le fait que l'on se trouve face à une certaine altérité, qu'il faut tenter d'appréhender, de comprendre, avec laquelle il faut amorcer un échange et un construit, cela tend alors à justifier une approche méthodologique particulière qui s'est souvent mise en place sur les terrains africains, et qui peut être vue comme un rapprochement avec l'anthropologie's. Cette approche est essentiellement d'ordre qualitative ${ }^{6}$, s'appuyant notamment sur des entretiens, des analyses de discours ou encore sur des méthodes d'observation, le plus souvent de type participante. Mais surtout, elle s'appuie sur des pratiques de terrain que l'on pourrait ici qualifier, d'un point de vue temporel, de "prolongées », et qui peuvent parfois aller selon les objectifs, les envies ou les capacités du chercheur, de plusieurs mois à plusieurs années ${ }^{7}$.

\footnotetext{
5 Ou l'ethnologie en fonction des écoles de pensée propres à chaque pays [Lombard, 2004].

6 Mentionnons néanmoins les grandes enquêtes quantitatives mises en place par l'IRD dans certains pays africains depuis les années 1980.

7 Un large panorama de ces méthodes d'enquête est résumé dans un récent ouvrage [2008] de Jean-Pierre Olivier de Sardan intitulé «La rigueur du qualitatif. Les contraintes empiriques de l'interprétation socioanthropologique ».
}

\section{La méthode anthropologique et ses difficultés d'application : le cas du Niger}

C'est dans cette démarche anthropologique que s'inscrivait notre projet de recherche $^{8}$ portant sur des dynamiques agricoles, foncières et migratoires. Celui-ci nous conduit au Niger en mars et avril 2010, où nous y avons fait l'expérience d'un travail de terrain rendu compliqué par une situation, puis un évènement.

Une situation, puisque nous nous rendions dans une zone déconseillée pour des raisons de sécurité, en lien avec la présence d'acteurs jugés dangereux. La zone correspondait à un territoire situé au Nord de la capitale Niamey, non loin de la frontière avec le Mali. Les acteurs dangereux étaient certains hommes armés participant à des mouvements de revendications, auxquels il faut ajouter des populations semi-nomades Touaregs en conflit ouvert avec des agriculteurs. Un évènement, car notre départ sur le terrain coïncidait, à quelques jours près, avec le renversement du président en place. Cela eut notamment pour conséquence la fermeture momentanée des frontières, ainsi que la fin des mandats des maires de communes, lesquels constituaient la seule autorité étatique présente dans la zone d'étude. Les conséquences de ce contexte difficile et de ces évènements ont ainsi perturbé notre approche du terrain de recherche. Ils contraignirent les déplacements, impliquèrent des adaptations méthodologiques et orientèrent même notre travail.

Nous avions initialement prévu de séjourner au sein de notre espace de recherche. Ce fut ici la première et majeure difficulté, puisque cela fut impossible. Sans autorisation de recherche, la situation en fut davantage compliquée. La solution fut d'effectuer des allers-retours entre la capitale et les villages étudiés, circulation compliquée par la présence de barrages militaires. Passer

\footnotetext{
8 Master 2.
} 
les différents barrages se faisait alors grâce à de l'argent distribué ou par le biais de la maitrise des lieux ${ }^{9}$ de nos connaissances, voir même grâce à l'aide de la participation des populations locales ${ }^{10}$. Cette situation de recherche changea naturellement la manière dont nous souhaitions procéder. Il y eu tout d'abord un temps accru consacré à certaines considérations logistiques ${ }^{11}$, et donc une réduction de celui consacré à l'objet de recherche en lui-même. Il fallut ainsi réinterroger notre notion du temps ${ }^{12}$ afin de concevoir sa malléabilité comme partie intégrante du travail de terrain.

Ensuite, l'impossibilité de séjourner dans les villages changea évidemment la manière dont nous avons pu collecter des données. Nous avions en effet initialement prévu d'aller séjourner au sein des villages étudiés, d'adopter ce que nous appellerons des grilles d'entretien souple, ou plus exactement des canevas d'entretien (Olivier De Sardan, 2008), avec pour volonté de rapprocher au maximum les entretiens de la discussion. Si nous avons eu parfois l'occasion de le faire, le peu de temps dont nous disposions une fois dans la zone nous rappelait à l'ordre ; et les grilles d'entretiens initiales, structurées, calées, pour ne pas dire rigides, furent à maintes reprises ressorties. Enfin, le temps de l'observation a inévitablement été réduit dans le temps. Paradoxalement, au sein de ce temps réduit, son rôle fut accru ${ }^{13}$.

Dans ce type de situations, certaines difficultés peuvent ainsi amener le chercheur à se considérer en "terrain sensible", notamment de par l'accès difficile à une zone d'étude théoriquement déconseillée. Mais cette situation montre également que le

\footnotetext{
${ }^{9}$ Contournement.

${ }^{10}$ Lesquels nous aidaient à nous fondre dans la masse des taxis brousse.

${ }^{11}$ Comment passe-t-on les barrages? Quelles sont les horaires à privilégier? Sur qui peut-on compter tel ou tel jour? Etc.

${ }^{12}$ Laquelle peut différée d'un continent à l'autre.

${ }^{13}$ Notamment dans la phase de repérage des localités à étudier.
}

chercheur n'est que rarement seul, et que toute entreprise d'aide ou de participation envisagée par d'autres acteurs à son égard participe également d'une autre mise en «danger", celle d'autrui. A quel point la pratique d'un «terrain sensible» par le chercheur participe-t-elle à la mise en «danger» de ses accompagnateurs et interlocuteurs? L'engagement sur le terrain peut-il faire abstraction d'un certain sentiment de culpabilité ? La décision de travailler en «terrain sensible » n'est-elle pas inéluctablement vouée à participer d'une mise en danger collective ? Si le chercheur, de par sa propre décision et volonté, peut se sentir dans une situation d'inconfort, comment appréhender ce même sentiment d'inconfort chez ceux qui pourtant sont dans leur propre pays?

Comme de nombreux autres travaux effectués sur des terrains sensibles, cet exemple soulève une question essentielle relative à la pertinence des données collectées, et plus globalement à la pertinence de travailler dans ces conditions. C'est notamment une interrogation qui a pu être formulée par un auteur comme Didier Fassin [2006] qui utilise l'expression d' "anthropologie embarquée», comme peut par exemple l'être le journalisme. Où se situe la limite au-delà de laquelle il n'est plus pertinent d'effectuer une recherche? Existet-il un stade où l'on doit juger que la recherche ne vaut plus la peine d'être poursuivie? Au contraire, doit-on effectuer son travail de terrain jusqu'au bout, quelle que soit l'ampleur des difficultés et des risques pris tant par soi que par les autres, quelle que soit la qualité des données collectées?

\section{Terrain sensible ou terrain miné ? Terrain perdu...}

Au vu des considérations évoquées cidessus, faut-il alors continuer à travailler sur les «terrains sensibles»? Tout d'abord, chaque "sensibilité» de terrain peut être jugée comme plus ou moins singulière, et 
donc plus ou moins surmontable. Accéder à une zone dite "sensible» ne représentera pas le même obstacle pour un chercheur ayant déjà vécu sur son terrain de recherche que pour celui qui s'y rend pour la première fois. Cela renvoie ensuite avant tout aux motivations et envies propres à chacun, ainsi qu'au degré d'engagement et d'implication du chercheur. Il n'y a donc évidemment pas de réponse " universelle » à cette question. De plus, il existe des arguments, en faveur $\mathrm{du}$ travail en terrains sensibles et en son encontre. Si le chercheur peut par exemple avancer que les populations présentes en terrain sensible doivent être étudiées au même titre que toutes celles davantage accessibles, il peut lui être répondu qu'il lui arrive, de par sa seule présence, de placer ses collaborateurs dans une situation d'inconfort. Celle-ci peut alors varier selon les profils de ceux-ci. La fonction exercée par un interlocuteur peut par exemple se révéler tantôt facilitatrice, tantôt complexifiante : les conditions de recherche ne seront pas les mêmes selon que l'on soit accompagné par un membre d'une ONG ou par un journaliste au sein d'un camp de réfugiés.

De manière plus pragmatique, cela dépend également - et surtout - de la possibilité «technique » d'effectivement s'y rendre. La question ici posée n'est alors pertinente qu'à partir du moment où la réponse s'inscrit dans une certaine liberté de choix, ce qui n'est pas - ou plus - notre cas.

En effet, à la suite des évènements de septembre $2010^{14}$, puis de janvier $2011^{15}$, le Niger - et notamment sa zone Nord - a vu son accessibilité réduite par l'ambassade de France. Depuis ces dates, le niveau théorique d'insécurité a été rehaussé et le Niger ne bénéficie plus à ce jour de zones jugées sécurisées par le gouvernement français. Si nous avons pu voir que la notion de « terrain sensible» pouvait revêtir des situations

14 Enlèvements de sept employés de l'entreprise française Areva à Arlit.

15 Enlèvements et décès de deux français à Niamey. disparates et concerner d'autres acteurs que le seul chercheur, cette situation nous offre d'autres aspects de la notion, ceux relatifs à sa construction et à son institutionnalisation. Si le terrain peut être jugé par le chercheur, et ce de manière personnelle et ressentie, comme sensible, les institutions ont quant à elles le pouvoir de décréter, et parfois d'entretenir, cette sensibilité. La dimension institutionnelle, et plus globalement politique est alors interrogée. Elle l'est d'autant plus que les niveaux de restriction émis peuvent diverger selon les États qui les façonnent, et les recommandations différer selon que l'on soit de nationalité française, anglaise ou américaine. Au regard de certains enjeux énergétiques de la France au Niger, la construction est ainsi naturellement politisée. Dans un récent document, Mehdi Taje ${ }^{16}$ nous rappelle que l'insécurité surmédiatisée reflétée par la série d'enlèvements d'Occidentaux et la montée en puissance du trafic de drogue doit être analysée avec prudence et remise en perspective par rapport aux stratégies des puissances étrangères (Taje, 2010). Que les restrictions soient ou non légitimes, la sensibilité du terrain peut varier selon l'intensité des enjeux, et le chercheur y est subordonné. Comment doit-être alors considéré le Niger aujourd'hui ? Comme un terrain sensible ou un terrain miné ? Un terrain sensible ne laisserait-il pas une certaine marge de manœuvre tandis que le terrain miné tendrait à la réduire à peau de chagrin ? Si tel est le cas, le Niger semble alors aujourd'hui un terrain tant miné que perdu; perdu pour le chercheur, miné par qui ?

Enfin, nous pensons que les difficultés de terrain parfois rencontrées peuvent avoir des creusets historiques. Si «nul n'est responsable des actes de ses ancêtres» (Pourtier, 2006 : 219), il n'en reste pas moins que les difficultés s'expriment aujourd'hui dans un contexte postcolonial caractérisé par des relations économiques encore proches de certaines logiques de domination. À la

${ }^{16}$ Géopoliticien, chargé des études africaines à l'Irsem (Ecole Militaire de Paris). 
question, la colonisation n'a-t-elle été qu'une simple parenthèse ou au contraire un moment essentiel dans l'histoire de l'Afrique, nous suivons le sillage d'Olivier De Sardan pour qui «la colonisation représente dans l'bistoire des peuples africains une telle rupture qu'il est impossible de ne pas distinguer un "avant " et un 《après » la conquête française » (1984: 5). Nous pensons que l'analyse du caractère « sensible » ou «miné » de certains lieux ne peut faire l'impasse de cette mise en perspective historique. Le chercheur originaire d'un pays de l'hémisphère Nord emporte et véhicule avec lui une image qui ne peut totalement s'évaporer après seulement un demi-siècle d'indépendance, il doit composer avec le regard qui lui est porté et garder omniprésente à portée de main toute sa réflexivité. L'histoire, mais également certaines dimensions géopolitiques ainsi que les politiques étrangères de chaque pays sont des éléments qui peuvent influer sur la sensibilité d'un terrain, et par conséquent sur la posture adoptée par le chercheur, laquelle doit découler de la connaissance de cet état de fait.

\section{« Glissement de terrain » et identité disciplinaire}

Voici une situation peu commode que de voir son terrain se dérober. En recherche d'adaptation, par quelle réflexion est-on guidé? Quels sont les différents déterminants qui peuvent conditionner la forme du rebond? Dans notre cas, nous pensons que la manière de s'adapter à la fermeture d'un terrain dépend de la manière dont celui-ci est perçu au sein d'une démarche de recherche, de la signification qui lui est accordé. La réorientation peut alors se faire sous influence affective et/ou identitaire.

Affective, voire émotionnelle, car un terrain de recherche n'est que rarement choisi au hasard. Les envies, les intérêts, ou encore l'histoire personnelle du chercheur sont des éléments affectifs parmi d'autres influençant le choix. Une fois le terrain perdu, c'est cette dimension affective qui est questionnée, renvoyant le chercheur à ses envies de recherche profondes et à ses questions fondatrices: pourquoi avionsnous initialement choisi ce lieu ? Pourquoi la démarche anthropologique? Passée la phase de déception et la question du "pourquoi », la réflexion s'engageait sur celle du « comment». Comment ré-envisager la démarche de recherche? Quelle première entrée allions-nous prendre? Celle du lieu ou de l'objet? Cela, nous semble-t-il, nous renvoyait à deux formes d'identités possibles (lieu/objet).

D’une manière générale, un jeune géographe est souvent identifié par rapport à un espace, à son espace, c'est-à-dire au lieu qu'il connaît bien, que cela soit une région ou bien un pays. "Si le modèle de la thèse régionale est passé de mode, il subsiste encore cette façon si particulière en géographie de se nommer, de se présenter à travers un espace d'etude. Ce mode de présentation de soi, sans doute anecdotique, peut aussi être perçu comme un élément révélateur de l'émergence tardive d'un discours critique sur le rapport au terrain dans la géographie française » [Labussière, 2008 : 3]. Ceci est d'autant plus vrai quand il s'agit de «terrains lointains ». La bonne connaissance de son terrain est aujourd'hui une obligation dans la recherche de légitimité de ses énoncés au sein de la communauté scientifique, le géographe et son terrain pouvant alors apparaittre sous la figure du couple [Goeury, $2008: 1]$. Bien souvent, capital spatial rime alors avec capital scientifique.

Il est ici néanmoins possible de nuancer la fidélité de ce couple. D'une part, il existe des géographes qui peuvent être davantage spécialistes d'une thématique ou d'une méthode que d'un espace; d'autre part, les trois dimensions (espace, thématique, méthode) peuvent très bien s'articuler entre elles au sein d'une "identité géographe ». Les questions plus globalement posées sont alors celles relatives aux «espaces vécus»: sans eux, est-on encore 
géographe ? L'espace vécu peut-il être autre que l'espace terrestre? Un auteur comme Cristina d'Alessandro-Scarpari [2010] s'interroge par exemple sur la manière de pratiquer une géographie contemporaine «sans terrain », notamment grâce à Internet ou aux changements d'échelle en lien avec la mondialisation. Si à cette position peut être répondu que les manifestations les plus parlantes de cette même mondialisation peuvent se retrouver à l'échelle locale/micro, mais également que Internet peut être considéré comme une forme de terrain; il n'en reste pas moins qu'il ne s'agit plus d'un terrain parcouru avec ses pieds. Les caractéristiques du terrain et les manières de le pratiquer ne représentent-elles pas une des nouvelles lignes de démarcation au sein de la discipline, ainsi que des évolutions liées à celles de certains objets?

Pour notre part, nous avons décidé d'opérer un "glissement de terrain », puisque nous avons décidé de suivre le cours du fleuve Niger et de nous arrêter en territoire béninois. C'est donc, en accord avec la tradition géographique, le choix de l'espace qui fut privilégié. Nous sommes en effet restés au sein de la même aire culturelle ${ }^{17}$, avons conservé la dimension affective - qui dans notre cas nie les frontières nationales -, ainsi que les premières compétences linguistiques acquises.

Si la première volonté était donc celle du lieu avant celle de l'objet, les deux ont en fait évolué de manière parallèle. Les lieux nigériens et béninois présentant chacun leurs singularités, l'objet a inévitablement été influencé par le changement spatial, traduisant ainsi la singularité de chaque couple unissant un objet et un lieu. En effet, le changement d'espace, ainsi que sa pratique, a largement influencé le nouveau façonnement de l'objet, puisque nous avons pu découvrir d'autres enjeux relatifs ${ }^{18}$ à la singularité de notre nouvelle zone d'étude.

\footnotetext{
17 Zarma-Songhay-Dendi.

${ }_{18}$ Comme par exemple les migrations féminines.
}

A la question, fallait-il d'abord façonner l'objet de recherche ou choisir le lieu de cette même recherche, la réponse reste ambigüe. Si l'on tente de complexifier la question, nous pouvons nous interroger, dans une optique plus méthodologique, sur deux postures : trouver sa bonne place vis-àvis de son terrain pour construire son objet ou trouver son objet pour décider de sa place vis-à-vis du terrain?

\section{Conclusion}

Concernant la dimension «sensible » du terrain, si nous avons pu voir que ce caractère pouvait être polymorphe, nous avons également pu constater que le choix d'un chercheur de vouloir passer outre cette sensibilité possède des implications pour d'autres acteurs de la recherche. À travers cet effet miroir ${ }^{19}$ de la sensibilité, c'est alors tout autant la présence du chercheur que l'image qu'il véhicule qui se trouvent questionnées.

Du point de vue de l'adaptation, si nous convenons que la difficulté, pour le géographe, est une occasion d'interroger son identité, à travers la pratique du terrain et l'idée qu'il s'en fait, nous conclurons en disant que nous avons saisi l' «occasion» de cette négociation identitaire pour interroger notre couple. À l'aise avec l'idée qui consiste à se laisser imprégner par l'espace - non pas porter par son terrain puisque à connotation passive - nous avons laissé et donné pleinement au terrain son rôle d'orienteur, de dé-constructeur et de reconstructeur, puisque c'es durant sa pratique que l'objet fut découvert et façonné.

Mais voici un choix bien personnel. Il existe évidemment de nombreuses approches différentes, lesquelles sont finalement moins liées au terrain en luimême qu'au chercheur. Deux chercheurs travaillant sur un même espace peuvent

19 Le terrain peut ne devenir sensible pour les personnes «accompagnatrices » ou «accueillantes » que parce que le chercheur est lui-même présent. 
tisser des liens diamétralement opposés avec ce dernier, traduisant des sensibilités, des caractères et des envies singulières. Au-delà des questionnements qu'elle provoque, des réflexions qu'elle engage et des justifications qu'elle implique, cette diversité des approches et des significations, source de richesse de la discipline, n'interroge-t-elle pas plus profondément les frontières de cette dernière?

Mickaël Quintard Doctorant en Géographie Migrinter UMR 6588 CNRS / Université de Poitiers mickael.quintard@univ-poitiers.fr

\section{Bibliographie :}

Agier, Michel (dir.) (1997) Anthropologues en danger. L'engagement sur le terrain, Paris, JeanMichel Place, 125 p. (Les Cahiers de Gradhiva).

Alessandro-Scarpari, Cristina (2010) Terrains africains, de la dénonciation au militantisme, L'information géographique, $\mathrm{n}^{\circ} 1$, pp. 55-62.

Bouillon, Florence ; Fresia, Marie ; Tallio, Virginie (dir.) (2006) Terrains sensibles Expériences actuelles de l'anthropologie. Paris, Centre d'études africaines, 208 p. (Dossiers africains).

Fassin, Didier (2006) L'innocence perdue de l'anthropologie: remarques sur les terrains sensibles, in Bouillon, F. ; Fresia, M., Tallio, $\mathrm{V}$. (dir.), Terrains sensibles - Expériences actuelles de l'anthropologie, Paris, Centre d'études africaines, pp. 97-103, (Dossiers africains).

Calbérac, Yann (2007) Le terrain des géographes, entre tradition disciplinaire et légitimation du chercheur, Cabiers ADES, version 1, pp. 19-25.
Calbérac, Yann (2010) Terrains de géographes, géographes de terrains, Lyon, Université Lumière Lyon 2, UMR 5600 «Environnement, ville, société », 392 p. Th. Doct. : Géogr. : Lyon : 2010.

Collignon, Béatrice ; Retaillé, Denis (2010) Introduction, L'Information Géographique, $\mathrm{n}^{\circ} 1$, pp. 6-8.

Dionigi, Albera (2001) Terrains minés, Ethnologie française, vol. 31, pp. 5-13.

Goeury, David (2008) Ces terrains lointains. De la naissance des hétérotopies en géographie, Communication au Colloque " À travers l'espace de la méthode: les dimensions du terrain en géographie", 18-20 juin 2008, Arras, $10 \mathrm{p}$.

Labussière, Olivier (2008) Le terrain? C'est ce qui résiste, Communication an colloque " $A$ travers l'espace de la méthode: les dimensions du terrain en géographie ", 18-20 juin 2008, Arras, $14 \mathrm{p}$.

Lombard, Jacques (2004) Introduction à l'ethnologie, Paris, Armand Colin, 192 p. (Cursus).

Olivier De Sardan, Jean-Pierre (1984) Les sociétés Songhay-Zarma (Niger - Mali). Chefs, guerriers, esclaves, paysans..., Paris, Karthala, 299 p. (Hommes et Sociétés).

Pourtier, Rolland (2007) Le terrain pour les tropicalistes, Bulletin de l'Association des géographes français, vol. 84, n 4, pp. 437-445.

Pourtier, Rolland (2006) L'Afrique noire au crible de la mémoire coloniale, Hérodote, n¹20, pp. 215-230.

Quiton, Philippe (2002) Le sens du terrain, Etudes et communication, ${ }^{\circ} 25$, pp. 41-49.

Taje, Mehdi (2010) Vulnérabilités et facteurs d'insécurité an Sabel, Note CSAO/OCDE, Enjeux ouest-africains, 8 p. 